\section{A personality-based intervention moderately reduces risk of drinking in at-risk 13-14-year-old British school children}

\section{QUESTION}

Question: Can personality-targeted interventions delivered by teachers delay the uptake of drinking and reduce risk of alcohol-related problems?

Patients: 1159 adolescents in UK school year 9 (aged 13-14) with at-risk personality type for alcohol as determined by the Substance Use Risk Profile Scale.

Setting: Two London schools.

Intervention: Personality-specific intervention versus control conditions. The intervention consisted of two 90-min group sessions per participant carried out by trained facilitators and co-facilitators over a period of 4 months. The intervention incorporated psychoeducational, motivational enhancement therapy, cognitive-behavioural therapy components and sharing of real-life scenarios. All exercises were delivered by trained staff and were discussed in a personality-specific way, specifically relating to sensation seeking. The control group did not receive any additional input on top of statutory drug education which all participants received in class.

Outcomes: Primary outcomes were drinking and binge drinking rates. Drinking was assessed by asking students whether they had consumed alcohol in the previous 6 months while binge drinking was assessed by asking students whether they had consumed five or more alcoholic beverages (or four or more for girls) on one occasion in the previous 6 months. Other outcomes included a quantity by frequency score (product of quantity and frequency measures) of alcohol use and drink-related problems.
Patient follow-up: $87 \%$.

\section{METHODS}

Design: Randomised controlled trial.

Allocation: Unclear.

Blinding: Not blinded.

Follow-up period: Six months.

\section{MAIN RESULTS}

Out of 2506 adolescents whose response to a screening survey was analysed, 1159 were considered to be an 'at risk' group and were randomised to intervention or control. Forty-one per cent of adolescents who were considered at risk were drinkers and $22 \%$ were binge-drinkers. The intervention group were 0.6 times less likely to report alcohol use than the control group (OR $0.6,95 \%$ CI 0.4 to $0.8, p<0.01$ ). The intervention did not affect the likelihood of binge drinking in the group as a whole but reduced the likelihood in adolescents who were alcohol users at study entry (OR $0.45,95 \%$ CI 0.3 to 0.8 ). High-risk adolescents reported lower quantity by frequency of alcohol use $(\beta=-0.18)$ and drinking-related problems $(\beta=-0.15) \mathrm{com}$ pared with the non-treatment group at follow-up.

\section{CONCLUSIONS}

In 13-14-year olds at risk of drinking, a personality-targeted intervention can decrease their likelihood of drinking. The intervention lowers the risk of binge drinking only in those who are already drinkers at the start.

\section{ABSTRACTED FROM}

O'Leary-Barrett M, Mackie CJ, Castellanos-Ryan N, et al. Personality-targeted interventions delay uptake of drinking and decrease risk of alcohol-related problems when delivered by teachers. J Am Acad Child Adolesc Psychiatry 2010;49:954-63.e1.

Correspondence to: Patricia Conrod, Institute of psychiatry, King's College London, 4 Windsor Walk, Denmark Hill, London SE5 8AF, UK;

patricia.conrol@kcl.ac.uk

Source of funding: Action on Addiction.
0 'Leary-Barrett and colleagues correctly note that universal alcohol and drug prevention programs have often been shown to be ineffective among adolescents, suggesting the need for alternative strategies. Among older adolescents and young adults a number of studies have shown that brief interventions conceptually similar to the authors' Preventure Programme are effective at reducing alcohol misuse among at-risk drinkers. ${ }^{1}$ The findings from the current study suggest that the benefits of these types of targeted interventions also extend to younger adolescents.

Perhaps the most important finding from the study was that the Preventure Programme intervention could be effectively delivered by teachers, as opposed to trained clinicians hired specifically for the study. Brief alcohol and drug prevention interventions have often been evaluated only in the context of an investigator's laboratory setting, thus they do not tell us how well an intervention may transfer to a real-world setting like the classroom or healthcare centre. The promising findings suggest that the Preventure Programme intervention can be effectively disseminated, although replication students are warranted.

One important unanswered question about the intervention is exactly why it works. The assumption seems to be that the intervention is effective because it is targeted to a self-reported dominant personality variable like hopelessness or impulsivity, but the study does not provide any evidence that this is in fact the case. In order to assess the validity of the proposed mechanisms of change, it will be important for researchers to evaluate whether personality-specific variables mediate intervention effects on relevant outcomes. Otherwise, it is difficult to argue that targeting the intervention based on personality characteristics is crucial to its success. For example, researchers should be able to identify mediators that, theoretically, change as a result of the intervention, which in turn impact drinking-related outcomes leg, depressive or hopelessness symptoms for those receiving the hopelessness-targeted intervention; self-control strategies for those receiving the impulsivity-targeted intervention). Identifying such mediators would provide both theoretical support for the intervention and suggestions for future clinical refinements.

\section{Matthew P Martens}

University of Missouri, Columbia, Missouri, USA

Competing interests None.

\section{REFERENCE}

1. Larimer ME, Cronce JM. Identification, prevention and treatment revisited: Individual-focused college drinking prevention strategies 1999-2006. Addict Behav 2007;32:2439-68. 